\title{
One health and One medicine: A review of the literature
}

\author{
Wubshet Asnake Metekia a*, Beyza H. Ulusoy ${ }^{b}$, Canan Hecer ${ }^{c}$ \\ $a, b, c$ Near East University, Faculty of Veterinary Medicine, Department of Food Hygiene and Technology, Nicosia, TRNC \\ * Corresponding author: Wubshet Asnake Metekia (wublivelygib@gmail.com)
}

How to cite this article: Metekia et al. One Health and One Medicine: A Review of the Literature. Veterinary Medicine and Public Health Journal 1(3); 2020: 91-95.

DOI: https://doi.org/10.31559/vmph2020.1.3.4

Received Date: 3/5/2020

Accepted Date: 15/6/2020

\begin{abstract}
The interface between human and animal is recognized as a critical point where zoonotic diseases can emerge and reemerge. These are majorly aggravated by globalization, urbanization, and trade of animals and animal byproducts etc. Infectious diseases can therefore occur at the border of human, animal and ecosystem interaction. In this context, health has been viewed and defined by most scholarls at three angles; i.e. at individual level, population or group level and the ecosystem level. In line with this, the one health approach considers human, animal and environmental health, and currently it is worldwide recognized approach. One health considers so many fields, due to this it is known as multidisciplinary approach, and promotes good health for all creatures (humans, animals and the surrounding ecosystem) because one cannot live alone without the interaction of another. So, the purpose of this review is to avail current scientific information and areas of cooperation between the two medicines, so as to take action in the one health and one medicine agenda. Generally, health for all motto is recognized throughout the world even if it requires multisectoral unity and collaboration, responsible team and/or institutional rearrangement, planning and prioritization of issues. Finally, for effective implementation there should be strong innovative biomedical research, policy and institutional setting, university curricula rearrangement, continuous advocacy, awareness creation and education of community and implementers.
\end{abstract}

Keywords: Human medicine; veterinary medicine; zoonotic diseases; one health and one medicine.

\section{Introduction}

The interface between human and animal is recognized as a critical point where zoonotic diseases can emerge and reemerge. This has been aggravated by globalization, urbanization, and trade of, particularly live animals and their products. Additionally, water and environmental pollution and climate change, loss of wild animal habitat, deforestation, wild animal hunting and consumption, modern intensive farming technologies and practices including the use of animal manure for fertilizer, and extensive use of pesticides and insect-sides have all been viewed as important risk factors for the emerging and reemerging of diseases. Hence, infectious diseases can occur at the border of human, animal and ecosystem interaction (Pieracci et al., 2016; WHO 2005).
Experiences gained from past zoonotic disease outbreaks such as avian influenza (HPAI), pandemic influenza (H1N1), and severe acute respiratory syndrome (SARS) showed that new approaches are need at the human-animalecosystem boundary, to improve early diagnosis, surveillance, monitoring, prevention and control of infectious diseases and at the same time to reduce the public animal health risks from those and other zoonotic diseases (WHO 2005).

Hence, the one health approach plays a key role in the prevention and control of infectious diseases. According to WHO (2005) and Graham et al., (2008) reported 75 percent of the human diseases are infectious i.e. they easily transmitted from animals to humans and are known as zoonotic. To solve the problem, the one health principles dregs different studies from different dimension and discipline and then put together for better cooperation and union. In the case of the working environment, the one 
health approach may work interdisciplinary because of the working environment for animal and human is different but a better cooperation and implementation of one health solves this problem by considering minor differences. The purpose of this analysis is to avail current scientific information on the subject matter and to show areas of cooperation between the two medicines so as to take action in the one health and one medicine agenda.

\section{The concept of health}

Most of the researchers look the thought and definition of health at three angles; i.e. at individual level, population or group level and the ecosystem (environment) level (Lemer and Berg 2015). Firstly, two thoughts are used i.e. animal health and human health. Still there is a dialogue both in animal discipline and medicine on how to look animals. The name non-human animals referring to all animal species except mankind or humans has been used for many years (Bekoff 1994; Hillier 2017) and also commonly used in our daily work, researcher, science writer and also animal welfare promoters. Generally, animals show a huge variety of life forms and to lump them together as a unity seems questionable (Derrida 2008; Lerner 2008). So, another question which should be answered by one health is the definition of health which governs both animals and humans.

On the second level, let us see the definition of health at population level, here at least two things are considered; in human medicine one can may use public health where as in veterinary medicine and biology we commonly used flock/herd/group health or population health, however in this case we used population health in spite of the species involved (Lemer and Berg 2015).

Thirdly, at the ecosystem health level, three aspects will be considered (Lemer and Berg 2015)

- Individual level health which includes animal and human health,

- Population level health encompasses population health and community/public health,

- Ecosystem level health (environmental/ecosystem health)

According to many researchers, the concept of both human health and animal health ranges from simply physiological interpretation to more integrated holistic wellbeing including mind health and/or social well-being (Nordenfelt 2006; Lerner 2008; Gunnarsson 2006). Therefore, there is high similarity in the categories of health between the two fields.

In many circumstances, the health of the people, animal as well as the environment can be affected and/or disturbed by emerging and infectious diseases. Emerging infectious diseases are those zoonotic diseases that are well known, either or a new generic variant emergence or previously occurred strain type gains the potential for novel/increased morbidity. For example, there are different influenza viruses with different strains
(H1N1, H2N2, H5N1, H7N9 etc.), the recent Ebola virus and others are particular examples for this issue (Maslow 2017). This is provoked by the interaction and link of human-animal and the ecosystem; hence zoonotic infectious disease will emerge and/or re-emerged (Maslow 2017; Van Doorn 2017). According to Van Doorn (2017), over $73 \%$ of emerging and reemerging diseases are zoonotic; of which $60 \%$ requires an animal to continue existence and later zoonotically transmitted to humans. Another 5 to $10 \%$ of the pathogens are environmentally transmitted to humans and the remaining percentage are human to human transmited signifying that the interface (human-animal) is a risk for emergency. The relationship between human and animal health and veterinary medicine duties is clearly indicated by Pappiaoanou (2004) who suggested those disciplines should engaging in identifying risk factors for preventing and controlling of those diseases which are cause of death for millions of humans and animals across the world. The world has gained experience from past outbreaks such as Ebola virus in central Africa, avian influenza virus in Hong Kong (Asia) and west Nilevirus in USA and others. Consequently, the international health organizations and professionals well recognize and proved that zoonotic diseases are real threat to both humans and animals (Maslow 2017; Girma et al. 2014). Those events opened a new window for veterinary medicine to participate actively in prevention and control of those zoonotic diseases (Pappiaoanou 2004).

\section{One medicine}

By definition, one medicine is the earliest term used to indicate one health (www.onehealthintiative.com). Health scientists (both veterinary and human) are working in collaborative research in developing common medical treatments for zoonotic diseases. For example, at Michigan State University, Collage of Veterinary Medicine, in the comparative ophthalmology laboratory, they study inborn eye diseases in dogs and cats that also affect human beings, allowing them to extrapolate across species. This helps them to learn more about the process of those common diseases and see how different treatments work in animals to understand how they are likely to work in people. So, they can help people by helping animals (https://cvm.msu.edu/vetschooltails/one-medicine-to-treat-all).

An American scientist; the father of modern epidemiology, Dr. Calvin Schwabe (1927-2006) coined the term one medicine, it is a technical term and direction to the science of health and diseases without the consideration of difference between human and animal and he proposed unified medical/veterinary approach to zoonotic disease (Hristovski et al. 2010). Even if this thought is popularized, the one medicine currently is not applicable and both fields grown separately i.e. the human medicine and animal medicine separately developed and used because of the research, thinking, and market demand, population growth 
and urbanization and others (World Veterinary Congress, 2008).

There are so many common basic activities of modern medicine whether veterinary medicine or human medicine, starting from health extension, education and research to diseases diagnostic, surveillance, prevention and control to clinical service including vaccination and other services, so professionals and researchers should work and come up with possible applicable solution for this issues to implement in the ground (Zinsstag 2011; Mathias 1998).

\section{One health}

One health has so many definitions.The most accepted definition implies that the wellbeing of the people is attached to the health of the animals and the surrounding environment (ecosystem) (Gyorffy and Jozwiak 2016). It promotes good health for humans, animals and ecosystem. According to American Veterinary Medical Association (2008) final report, one health is the combined endeavor of multidiscipline working locally, nationally and globally to attain best health for people, animals and the ecosystem.

In the mid-1800s, a German scientist called Rudolf Virchow, for the first time he advocates one health and he said there is no dividing line between the two medicines i.e. human and veterinary medicine (www.cdc.gov/eid).

The one health approach considers human, animal and environmental health. In line with this, currently the one health approach is a worldwide recognized approach. By definition, one health encompasses so many fields like epidemiology, environmental sciences, medicine, toxicology, botany, zoology, ecology, sustainable agriculture, economics, anthropology, ethnography and other social sciences. Due to the above various scientific disciplines, one health pursuit's better health for humans, animals and the surrounding ecosystem and also one cannot live alone without the interaction of another like human being cannot live without the surrounding ecosystem and also animals cannot not live without the ecosystem, the activities and condition of one affect other on a variety of levels (economic, physical, social and more) (Schwab 2008; Gyorffy and Jozwiak 2016).

Correctly, most ecosystems are on alarming stage i.e. they are converted into different practices or uses like water pollution, watersheds degradation and deforestation, overgrazing etc. because of the high population growth and urbanization, resettlement, free animal grazing, construction of roads, free waste disposal etc.

A study conducted in Ethiopia indicates the significance of one health approach in the surveillances and diagnosis of zoonotic diseases by multispectral unity and collaboration i.e. from different offices (human health, animal health and environment) and the approach creates national and regional capacity through strengthening and upgrading of public health and veterinary laboratories, diseases surveillance, information sharing system, prevention and control (Pieracci et al. 2016). The American Veterinary Association and World Veterinary Congress (2008) highlighted that multiple disciples and institutional working locally, nationally and globally should work together in collaboration and synergistic effort to ensure good health for human, animal and the environment.

\section{The role of veterinarians in the one health agenda}

The veterinarians as a key professional in the one health agenda, control zoonotic and food born diseases, residual effects of various compounds/hormones that guarantee protected animal products and by products for human consumption as well as to advance the wellbeing of the public (Gyorffy and Jozwiak 2016). In addition, the veterinarian implements the one health concept in veterinary programs, they form alliance with other health workers and scientific communities. Not only this, but also, they facilitate and act to create good leadership, management skill, new policy issues development and they will design future direction in their subject area (Gyorffy and Jozwiak 2016; Schoemana et al. 2009; World Veterinary Association 2016; Wongand Kogan 2013). Fortunately, veterinarians take intensive training about comparative medicine, zoonosis, publıc health issues and also have better access to both in field and clinic level to critically observe animals, humans and their sounding ecosystem so that they are fully aware of the possible risk of zoonotic diseases both in human and animals and also they are responsible to detect, prevent and control those diseases. Physicians on the other hand, lack this thing, so veterinary medicine is in a better position to discover public health threats, establish surveillance and control systems than general health practitioners (American Veterinary Association and World Veterinary Congress 2008).

According to Gyorffy and Jozwiak 2016 study in USA, they critically illustrated by considering military veterinarian positioned in a military area where the next collaborator holding a medical degree is stationed hundreds or thousands of kilometers away. So military veterinarians are in the front line to combat (prevent and control) zoonotic diseases especially in military area, wildlife parks and remote areas. Therefore, one health can be better recognized by those professional military veterinarians for the better alignment of the two medicines.

\section{Association of one health and one medicine}

According to the American Veterinary Association and World Veterinary Congress (2008), FAO/OIE/WHO (2010) and one health initiatives (http://www.onehealthintiative.com/), the relationship and importance of one health and one medicine is promoted by many international, national and regional organizations and governments including AVMF, CDC, OIE, WHO, WVC, one health network, one health Initiative and others. Due to this, there are many bright plans, projects and ongoing researches here and there in the world. The association and importance of one health and one 
medicines are many, such as; (a) similar biology, both humans and animals are grouped under kingdom animally, (b) clinical experiments such as trials of new vaccines, trials of new drugs, nutritional choices, dietary supplements, trials of medical devices and procedures, (c) majority of infectious diseases are originated from animals thus recent investigations focus on the development of one medicine for both humans and animals in order to control and eradicate zoonotic diseases, (d) rule of one health and one medicine in education systems of universities to educate students on one health, (e) reduces duplicated efforts and promotes one finance/money system, one manpower, one laboratory set up and facilities, one samples to help focusing mutual benefits and fighting zoonotic diseases, so that we can build one world, one health and one medicine and impacting the global health (Pieracci et al. 2016; Lemer and Berg 2015; http://www.onehealthintiative.com/;

https://cvm.msu.edu/vetschool-tails/one-medicine-totreat-all).

\section{Conclusion}

Generally, one health and one medicine are the right solution for the current and complicated health problems of the world due to infectious diseases Health for all motto has been recognized throughout the world even if it requires multisectoral unity and collaboration, responsible institution/ team building, planning and prioritization of issues (diseases), resource mobilization, result based monitoring and evaluation and reporting to concerned bodies national, regional and to the global networks that work on this issue. Lastly, there should be strong innovative biomedical research (such as animal gene improvement, development of treatments, vaccines, theriogenology, food safety, food and nutrition, acute diseases, genetic improvement of animals, diagnostic tools and more), policy and institutional setting, university curricula rearrangement including incorporation of useful perspective and treatments from traditional medicine, continuous advocacy, awareness creation and education to the community and implementers should be done.

\section{References:}

[1] American Veterinary Association and World Veterinary Congress. (2008). "One world one health and one medicine".

[2] American Veterinary Medical Association. (2008). "One health a new professional imperative, one health initiative task force final report".

[3] Andrea. GYORFFY \& Akos. Jozwiak, (2016). "A Hidden Corner of the "One Health" Concept: One Health". the Military Veterinarian and Education.

[4] Bekoff. M. (1994). "Cognitive ethnology and the treatment of non-human animals: how matters of mind inform matters of welfare".

[5] Calvin Schwab, (2008) .one health institute Calvin Schwab project.

[6] Derrida J. (2008). "The Animal that therefore I am". Fordham university press. New York.
[7] Pieracci. Emily G. et al. (2016). "Prioritizing zoonotic diseases in Ethiopia through one health approach". One Health. 2: 131-135, https://doi.org/10.1016/j.onehlt.2016.09.001

[8] "Emerging Infectious Diseases" 14(9), 2008, www.cdc.gov/eid

[9] FAO/OİE/WHO. (2010). Joint scientific consultation. "Influenza and other emerging zoonotic diseases at the human animal interface". Italy.

[10] General Accounting office. (2000. "West Nile Virus Outbreak: Lesson to public health preparedness", report to congressional requests.

[11] Girma. Sisay et al. (2012) . "Assessment of awareness on food born zoonosis and it's relation with veterinary public health service in and around Addis Ababa". Journal of Public Health and Epidemiology. 4(2), https://doi.org/10.5897/jphe12.004

[12] Gunnarsson. S. (2006). "The conceptualization of health and diseases in veterinary medicine".

[13] Hillier. Jean. (2017). "No place to go? Management of non-human animals overflows in Australia, center for urban research school of global", urban and social studies, RMİT University, city campus, GOP Box 2476V, Melbourne, Victoria 3001, Australia.

[14] Hristovski. M, Cvetkovik. A, Cvetkovik. I, Dukoska. V. (2010). "Concept of one health - a New Professional Imperative". Maced J Med Sci. 3(3):229-232, doi.10.3889/MJMS.1957-5773.2010.0131.

[15] https://cvm.msu.edu/vetschool-tails/one-medicineto-treat-all, accessed on December 20, 2017.

[16] http://www.onehealthinitiative.com/publications, accessed on December 20, 2017.

[17] Joel N. Maslow. (2017). "Viccine development for emerging virulent infectious diseases", St. Georges University, United States.

[18] Lerner. H. (2008). "The concept of health, wellbeing and welfare as applied to animals. a philosophical analysis of the concepts with regard to difference between animals". PhD thesis Linkoping university.

[19] Lemer. Henrik \& Berg. Charlotte. (2015). "The concept of health in one health and some practical implications for research and education: what is one health? “. Infection Ecology \& Epidemiology. 5(1): 25300, https://doi.org/10.3402/iee.v5.25300

[20] Mathias, E. (1998). "Agriculture and human values" 15:145. https://doi.org/10.1023/A:1007439228438.

[21] Nordenfelt. L. (2006). Animal and human health welfare: a comparative philosophical analysis, Wallingford, UK.

[22] Pappiaoanou. M. (2004). "Veternary medicine protecting and promoting the public's health and wellbeing". office of Global health, Mailstop D-69, Center for disease control and prevention, Atlanta, GA 30333, USA.

[23] Rogier. H. \& Doorn Van. (2017). "Emerging infectious disease". Nuffield Department of Medicine. university of oxford, UK.

[24] Schoemana. J P et al. (2009). "A case study based, small group cooperatives learning courses in preclinical veterinary science aimed at bridging basic science and clinical literacy. Journal of the South African Veterinary Association. 80(1), https://doi.org/10.4102/jsava.v80i1.165

[25] Taylor. H., Sophia. M., Latham. Mark E. J. Woolhouse. (2001). "Risk factors for human diseases emergence", center for tropical veterinary medicine, University of Edinburgh, Eastr Bush, Roslin, Midlthian, EH 25 9RG, UK.

[26] WHO. (2005). "Combating emerging infectious diseases in the south East Asia region". 
[27] Wong. D. \& Kogan. L.R. (2013). 'Veterinary students' attitudes on one health: implications for curricula development at veterinary collages". Journal of veterinary medical education. 40 (1): 58:62, https://doi.org/10.3138/jvme.0612.057r

[28] World Veterinary Congress. (2008). "One world one health and one medicine".
[29] World Veterinary Association. (2016). "World veterinary association position on one health concept".

[30] Zinsstag. J, Schelling. E, Waltner -Toews. D. \& Tanner. M. (2011) "from one medicine to one health and systematic approaches to health and well-being". Preventive veterinary medicine". 101(3-4):148-156. https://doi.org/10.1016/j.prevetmed.2010.07.003 